\title{
The Relationship Between Chronic Kidney Disease and the Severity and Long-Term Prognosis of Patients with Coronary Artery Disease After Drug-Eluting Stent Implantation
}

This article was published in the following Dove Press journal:

International Journal of General Medicine

\author{
Xianjing Wei ${ }^{1}$ \\ Ying Zhang' \\ Gaoliang Yan ${ }^{2}$ \\ Xiaoqing Wang' \\ 'Department of Cardiology, The \\ Affiliated Zhongshan Hospital of Dalian \\ University, Dalian, Liaoning, I I00I6, \\ People's Republic of China; ${ }^{2}$ Department \\ of Cardiology, Zhongda Hospital of \\ Southeast University Medical School, \\ Nanjing, Jiangsu, People's Republic of \\ China
}

\begin{abstract}
Objective: To investigate the relationship between chronic kidney disease (CKD) and the severity and long-term prognosis of patients with coronary artery disease (CAD) after drugeluting stent (DES) implantation.

Methods: There were 814 patients, who consecutively received a DES implantation, selected for this study. They were divided into two groups, according to whether or not they suffered CKD. There were 254 cases in the CKD group (31.2\%), while there were 560 cases $(68.8 \%)$ in the control group. The clinical characteristics, coronary artery lesions, and major adverse cardiac and cerebrovascular events (MACCE) of the two groups were compared, and the relationship between risk factors and MACCEs was analyzed by multivariate logistic regression.
\end{abstract}

Results: Compared with the control group, the CKD group had more severe coronary artery stenosis, expressed as the more diseased arteries $(2.15 \pm 0.82$ vs $1.87 \pm 0.83, p=0.001)$, a high incidence of three diseased arteries $(42.0 \%$ vs $28.3 \%, p=0.001)$, and a higher Gensini score [37 $(18.6,66)$ vs $27.5(12,52.5), p=0.009]$. The one-year post-implant incidence of MACCE was higher in the CKD group compared with the control group ( $17.6 \%$ vs $9.9 \%, p=0.006)$.

Conclusion: CKD appears to be an important predictor for the prognosis of CAD.

Keywords: chronic kidney disease, coronary artery disease, Gensini score, major adverse cardiac and cerebrovascular events

\section{Introduction}

In recent years, more and more attention has been paid to the occurrence and development of chronic kidney disease (CKD) alongside coronary artery disease (CAD). ${ }^{1,2}$ The relationship between the two diseases is complex. It has been agreed that the incidence and mortality of CAD increase significantly in patients with CKD, especially in patients with end-stage renal disease. ${ }^{3-5}$ Studies have also shown that the incidence of CAD, left ventricular hypertrophy, chronic heart failure, and stroke is significantly increased in patients with mild chronic renal dysfunction without any traditional risk factors for coronary heart disease. Therefore, the risk of cardiovascular disease in patients with CKD may be more serious and occur earlier than previously recognized. Even mild renal impairment should be considered as a high-risk factor for coronary heart disease. ${ }^{6}$ However, how CKD affects the severity and prognosis of coronary artery stenosis in coronary heart disease has
Correspondence: Xianjing Wei

Department of Cardiology, The Affiliated Zhongshan Hospital of Dalian University, No. 6 Jiefang Street, Zhongshan District, Dalian City, Liaoning Province, II0016,

People's Republic of China

Tel +8618642668608

Email weixian_jing@163.com 
not been reported in large sample sizes. The purpose of this study was to investigate the effects of CKD on coronary stenosis and drug-eluting stent implantation in patients with CAD by analyzing their clinical and followup data.

\section{Methods}

Between January 2015 and December 2017, 814 patients with coronary heart disease who underwent drug-eluting stent implantation for the first time in the Department of Cardiology of our hospital were enrolled in the study. Exclusion criteria: (1) patients who had received previous coronary interventions or coronary artery bypass surgery; (2) patients who had both bare-metal stents and drug-eluting stents implanted; (3) patients with acute renal insufficiency or CKD requiring long-term regular hemodialysis and peritoneal dialysis; (4) patients with acute coronary syndrome requiring emergency coronary intervention therapy; and (5) patients whose medical records were incomplete. The ethics committee of the Cardiovascular Institute granted ethical permission for this study, and all the patients provided written informed consent. The drug-eluting stents used in this study were obtained from Lepu Medical Technology (Beijing, China), MicroPort Scientific Corporation (Shanghai, China), JW Medical Systems (Weihai, Shandong, China), Abbott (Chicago, IL, USA), Boston Science (Boston, Massachusetts, USA), and Medtronic (Minneapolis, Minnesota, USA).

Data collection involved the retrospective analysis of patients' clinical data and detailed records of patients' medical history, symptoms, signs, and test results. The glomerular rate filtration (eGFR) was estimated according to an improved Modification of Diet in Renal Disease (MDRD) formula for Chinese patients: c-aGFR 1 (mL/min per $\left.1.73 \mathrm{~m}^{2}\right)=186 \times \mathrm{Pcr}^{-1.154} \times$ age $^{-0.203} \times 0.742$ (if female) $\times 1.227$ (if Chinese), ${ }^{7}$ which had small deviation and good accuracy. The eGFR $<90 \mathrm{~mL} /\left(\min 1.73 \mathrm{~m}^{2}\right)$ was used as the criterion of chronic renal dysfunction. The study intended to analyze the characteristics of patients with coronary heart disease complicated with chronic renal insufficiency. Body mass index $=$ body weight $(\mathrm{kg}) /$ height $(\mathrm{m})^{2}$.

The diagnostic criteria for coronary heart disease involve the main coronary arteries, including the left main artery, the left anterior descending artery, the left circumflex artery, and the right coronary artery. CAD is diagnosed when the stenosis of any main coronary artery is more than $50 \%$. Coronary angiography of the patients in the study showed that lesions with a stenosis of lumen diameter of more than $50 \%$ involved a number of branches of the left anterior descending, left circumflex, or right coronary artery. They were divided into one, two, and three lesion groups (two lesions involving the left anterior descending branch and left circumflex branch).

The degree of coronary artery stenosis was assessed quantitatively by the Gensini integral system; ${ }^{8}$ coronary artery stenosis $<25 \%$ scored 1 point, 26-50\% scored 2 points, $51-75 \%$ scored 4 points, $76-90 \%$ scored 8 points, $91-99 \%$ scored 16 points, and 100\% scored 32 points. Different segments of coronary artery stenosis were multiplied by corresponding coefficients, according to Gensini scoring standards, and the coronary artery stenosis score of each patient was the sum of the scores of each branch.

The criterion for determining stroke and peripheral artery disease is stroke history, defined as a history of ischemic cerebral infarction or hemorrhagic cerebrovascular accidents. A history of peripheral arterial disease is defined as previous atherosclerotic arterial diseases of the upper or lower extremities, including atherosclerotic occlusion or stenosis confirmed by ultrasonography.

Follow-up was undertaken by telephone or an outpatient visit, while a major composite endpoint event may have led to re-hospitalization. The incidence of major cardiovascular and cerebrovascular adverse events (MACCE) during hospitalization and within one year after discharge was recorded. MACCE is defined as a composite endpoint of all-cause death, non-fatal myocardial infarction, non-fatal stroke (transient ischemic attack, cerebral infarction, cerebral hemorrhage), or revascularization (thrombolysis, stenting, bypass) events.

SPSS 19.0 statistical software (International Business Machines Corporation, Armonk, New York, USA) was used for statistical analysis. The measurement data were expressed by mean + standard deviation, and the comparison between groups was expressed by independent sample t-tests. The counting data were expressed by percentage, and the comparison between groups was tested by Chi-squared analysis. The cumulative survival rate was analyzed by the Kaplan-Meier method. The survival rate curve of Kaplan-Meier was tested by the log-rank method. At the same time, using MACCE as dependent variables and related clinical factors as co-variants, logistic regression analysis was conducted to determine the independent risk factors affecting clinical prognosis. $p<0$. 05 was statistically significant. 


\section{Results}

\section{General Information}

There were 814 patients who met the inclusion criteria, comprising 584 men (71.7\%) and 230 women (28.3\%) with an average age of $63.3+10.9$ years. The patients were divided into two groups according to whether they had CKD. There were 560 cases $(68.8 \%)$ of CAD combined with CKD and 254 cases (31.2\%) of CAD alone.

\section{Baseline Data of Patients with Coronary Heart Disease in CKD Group}

As shown in Table 1, there were no significant differences in the incidence of gender, smoking history, diabetes, and obesity between patients with and without CKD. When comparing the two groups, the CKD group was older and had a higher proportion of hypertension, hyperlipidemia, and a history of cerebrovascular disease. There were no significant differences in fasting blood glucose, triglyceride, total cholesterol, and low-density lipoprotein levels.

\section{Comparison of Stenosis Degree of CAD Between Patients with and without CKD}

Compared with patients without CKD, patients with CKD had more severe stenosis of coronary artery lesions (2.15+
0.82 vs $1.87+0.83, p=0.001$ ), a higher proportion of three-vessel lesions $(42.0 \%$ vs $28.3 \%, p=0.001)$ and higher Gensini scores (see Table 2).

\section{Comparison of Prognosis After Drug-Eluting Stent Implantation in Patients with and without CKD}

As of January 2019, there was a one-year follow-up period for all patients, with a total of 43 cases lost. Among them, 31 cases were lost in the combined CKD group, and 12 cases were lost in the non-combined CKD group. A total of 771 patients were followed up, giving a study follow-up rate of $94.7 \%$ (771/814). During the follow-up period, 93 patients with CKD had MACCE events, the incidence of MACCE being 17.6\% (93/529), while 24 patients without CKD had MACCE events, the incidence being 9.9\% (24/ 242). The cumulative incidence of MACCE in the CKD group was significantly higher than that in the non-CKD group $(17.6 \%$ vs $9.9 \%, p=0.006)$ (see Figure 1$)$. There was no significant difference in all-cause mortality between the two groups ( $p=0.578,2.3 \%$ vs $1.7 \%$ ), but the incidence of non-fatal myocardial infarction $(p=$ $0.034,6.6 \%$ vs $2.9 \%$ ), non-fatal stroke ( $p=0.043,7.0 \%$ vs $3.3 \%$ ), and Tricuspid valve replacement $(p=0.003$,

Table I Clinical Baseline Comparison of Patients with and without CKD

\begin{tabular}{|l|c|c|c|}
\hline Factors & CKD Group & Non-CKD Group & p value \\
\hline Age (age) & $65.8 \pm 10.4$ & $57.63 \pm 10.0$ & $<0.00 I$ \\
Male (\%) & $398(71.1)$ & $186(73.2)$ & 0.557 \\
Smoking (\%) & $272(48.8)$ & $140(55.6)$ & 0.081 \\
Hypertension (\%) & $421(75.5)$ & $160(63.5)$ & $0.00 I$ \\
Hyperlipidemia (\%) & $353(63.8)$ & $142(56.1)$ & 0.043 \\
Diabetes mellitus (\%) & $185(33.2)$ & $70(27.6)$ & 0.121 \\
History of cerebrovascular diseases (\%) & $93(16.8)$ & $22(8.7)$ & 0.002 \\
Obesity (\%) & $114(22.7)$ & $46(21.0)$ & 0.697 \\
Fasting blood glucose (mmol/L) & $5.59 \pm 1.46$ & $5.77 \pm 1.69$ & 0.115 \\
Triglyceride (mmol/L) & $1.60 \pm 0.87$ & $1.54 \pm 1.12$ & 0.455 \\
Total cholesterol (mmol/L) & $4.62 \pm 0.97$ & $4.62 \pm 0.92$ & 0.924 \\
LDL-C (mmol/L) & $2.83 \pm 0.79$ & $2.73 \pm 0.77$ & 0.122 \\
\hline
\end{tabular}

Abbreviation: LDL-C, low-density lipoprotein cholesterol.

Table 2 Comparison of Severity of Coronary Stenosis in Patients with and without CKD

\begin{tabular}{|l|c|c|c|}
\hline Factors & CKD Group & Non-CKD Group & $\boldsymbol{p}$ value \\
\hline Number of lesions & $2.15 \pm 0.82$ & $1.87 \pm 0.83$ & 0.001 \\
Three vessel lesions (\%) & $235(42.0)$ & $72(28.3)$ & 0.001 \\
Gensini score & $37(18.6,66)$ & $27.5(12,52.5)$ & 0.001 \\
\hline
\end{tabular}




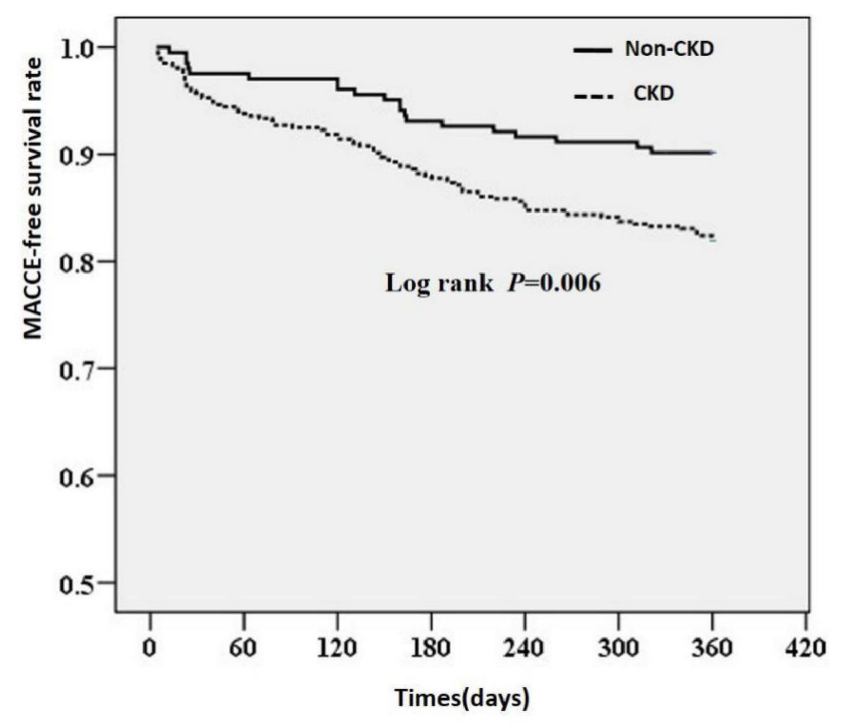

Figure I Prognostic comparison of the incidence of MACCE after drug-eluting stent implantation in patients with and without CKD.

$13.1 \%$ vs $5.8 \%$ ) was significantly higher in the combined CKD group than in the non-combined CKD group, as shown in Table 3.

\section{Multivariate Logistic Regression Analysis of MACCE Events}

Multivariate logistic regression analysis was used to determine the independent risk factors affecting the clinical prognosis by taking the occurrence of MACCE events (1 = yes, $0=$ no) as the dependent variable and related clinical factors as covariates. Logistic regression analysis showed that the history of CKD ( $p=0.046)$ was the relevant factor affecting the occurrence of MACCE (see Table 4 for details).

\section{Discussion}

The results of this study show that coronary artery lesions are more serious and the long-term prognosis is worse in

Table 3 MACCE Events After DESs Implantation in Patients with and without CKD

\begin{tabular}{|l|c|c|c|}
\hline Factors & CKD Group & Non-CKD Group & p value \\
\hline MACCE & $17.6 \%(93 / 529)$ & $9.9 \%(24 / 242)$ & 0.006 \\
All-cause death & $2.3 \%(12 / 529)$ & $1.7 \%(4 / 242)$ & 0.578 \\
Non-fatal MI & $6.6 \%(35 / 529)$ & $2.9 \%(7 / 242)$ & 0.034 \\
Non-fatal stroke & $7.0 \%(37 / 529)$ & $3.3 \%(8 / 242)$ & 0.043 \\
TVR & $13.1 \%(68 / 529)$ & $5.8 \%(14 / 242)$ & 0.003 \\
\hline
\end{tabular}

Abbreviations: MI, myocardial infarction; TVR, target vessel revascularization.
Table 4 Multivariate Logistic Regression Analysis of MACCE Events

\begin{tabular}{|l|c|c|c|}
\hline Factors & OR & $95 \%$ CI & P \\
\hline CKD & 1.673 & $1.010-2.773$ & 0.046 \\
Male & 0.823 & $0.496-1.364$ & 0.450 \\
Age & 1.033 & $1.011-1.056$ & 0.003 \\
SBP & 0.989 & $0.977-1.000$ & 0.054 \\
Smoking & 1.181 & $0.759-1.837$ & 0.460 \\
Hypertension & 1.101 & $0.694-1.747$ & 0.682 \\
Hyperlipidemia & 0.864 & $0.581-1.284$ & 0.470 \\
Diabetes mellitus & 1.234 & $0.782-1.947$ & 0.366 \\
History of cerebrovascular diseases & 1.010 & $0.593-1.719$ & 0.972 \\
Hemoglobin & 1.008 & $0.993-1.023$ & 0.314 \\
Serum Calcium & 2.700 & $0.556-13.097$ & 0.218 \\
Fasting blood glucose & 1.163 & $1.033-1.309$ & 0.012 \\
\hline
\end{tabular}

patients with CAD complicated by CKD. These results are similar to those reported in the past. ${ }^{9}$ These results suggest that CKD is an important predictor of major cardiovascular and cerebrovascular adverse events in patients with CAD after stent implantation.

Chronic renal insufficiency affects CAD and clinical prognosis in many ways. Patients with renal impairment may be exposed to cardiovascular risk factors from the beginning of nephropathy. ${ }^{10}$ The possible mechanisms are as follows: ${ }^{11,12}$ (1) microinflammation: there is a general systemic microinflammation in patients with renal dysfunction - the essence of microinflammation is immune inflammation, while atherosclerosis is the microinflammation of the vascular wall; (2) oxidative stress; (3) endothelial dysfunction; (4) lipid metabolism disorder; (5) uremic toxins such as homocysteine, glycation end products, protein oxidation end products, parathyroid adenine, abnormal calcium and phosphorus metabolism, asymmetric dimethylarginine, hyperuricemia, and other factors that can cause vascular endothelial damage can be increased in patients with chronic renal insufficiency; (6) renin-angiotensin-aldosterone system activation and hypertension. Researchers have proposed that CKD and CAD share many common traditional risk factors, such as hypertension, diabetes, dyslipidemia, and many other common non-traditional risk factors, such as homocysteinemia, calcium and phosphorus metabolic disorders, elevated serum uric acid levels, elevated C-reactive protein, and other oxidative stress and inflammatory markers. ${ }^{13}$ It is generally believed that the presence of multiple risk factors will lead to superimposed or multiplied effects, which promote the development of vascular endothelial dysfunction and atherosclerosis. $^{12}$ 
A survey of 3513 patients with CAD showed that the incidence of CKD was as high as $24.8 \%(\mathrm{n}=871)$. Compared with patients without CKD, patients with CAD combined with CKD had a higher incidence of hypertension $(49.5 \%$ vs $42.8 \%)$. The results suggested that patients with CKD had a greater risk of CAD and a worse prognosis. ${ }^{14}$ Ryoo et al ${ }^{15}$ studied 53,221 Korean men. Framingham risk scores, which are divided into three levels: 10\% (low), 10-19\% (middle), and $>20 \%$ (high), were used to predict the probability of ten-year coronary events Multivariate logistic regression analysis showed that the risk of CAD was higher in patients with CKD, suggesting that $\mathrm{CKD}$ was independently related to the risk of CAD in Korean men.

Agrawal et al found that effective treatment for patients with acute and chronic CAD may have a limited effect on patients with chronic renal insufficiency. DES use reduced the risk for MACCE compared with BMS use with the CAD patients, in particular those with $\mathrm{CKD} .{ }^{16}$

In this study, patients with CAD complicated with renal insufficiency were followed up, and the proportion of adverse cardiovascular events were found to increase significantly. It has been seen that when coronary syndrome occurs in older patients with dysfunction or myocardial ischemia, CKD can lead to serious arrhythmias or other cardiac events, ${ }^{17}$ which is consistent with this study. Multivariate regression analysis showed that CKD was included. Renal function levels are related to the occurrence of cardiovascular adverse events during the follow-up period. Independent risk factors showed that Probably because of case selection, diabetes mellitus, and hypertension factors are excluded from the model, and the level of LDL-C may also be underestimated. Special Pathological Factors of Renal Insufficiency accelerated the deterioration of cardiovascular diseases, including calcium and phosphorus metabolic disorders that exacerbate vascular calcification, sympathetic nervous system long-term chronic activation and imbalance of vasoconstrictor factors of the system. ${ }^{18}$ Chronic renal insufficiency appears to be an important predictor of adverse cardiovascular events in older patients with coronary heart disease.

There are some limitations to this study. First, we conducted a single center retrospective observational study without long-term follow-up, which may affect the long-term prognosis of patients with coronary heart disease, and we were unable to fully assess the specific duration of CKD due to it being a retrospective study. Second, some CKD patients are classified only by a single EGFR measurement. The eGFR values were derived from individual serum creatinine levels measured before coronary angiography, and creatinine may be affected by drugs or acute clinical status. Third, we did not collect data on the course of renal function in these patients, whether before or after angiography.

\section{Conclusions}

In conclusion, our analysis of the clinical and follow-up date of a large group of patients with CAD after DES implantation shows that CKD was an important predictor for the prognosis of CAD in this population. Our study, therefore, suggests that it is important for physicians to implement early prevention and treatment strategies for patients with $\mathrm{CKD}$, with respect to the possible development of CAD.

\section{Ethics Approval and Consent to Participate}

This study was conducted with approval from the Ethics Committee of the Affiliated Zhongshan Hospital of Dalian University (2017024). This study was conducted in accordance with the declaration of Helsinki. Written informed consent was obtained from all participants.

\section{Acknowledgments}

We would like to acknowledge the hard and dedicated work of all the staff that implemented the intervention and evaluation components of the study.

\section{Funding}

No external funding received to conduct this study.

\section{Disclosure}

The authors declare that they have no competing interests.

\section{References}

1. Levey AS, Coresh J, Balk E, et al.; National Kidney Foundation. National Kidney Foundation practice guidelines for chronic kidney disease: evaluation, classification, and stratification. Ann Intern Med. 139;2003:137-147. doi:10.7326/0003-4819-139-2-200307150-00013

2. Wison S, Foo K, Cunningham J, et al. Renal function and risk stratification in acute coronary syndromes. $\mathrm{Am} J$ Cardiol. 2003;91:1051-1054. doi:10.1016/S0002-9149(03)00147-4

3. Sarnak MJ, Levey AS, Schoolwerth AC, et al.; American Heart Association Councils on Kidney in Cardiovascular Disease, High Blood Pressure Research, Clinical Cardiology, and Epidemiology and Prevention. Kidney disease as a risk factor for development of cardiovascular disease: a statement from the American Heart Association Councils on Kidney in Cardiovascular Disease, High Blood Pressure Research, Clinical Cardiology, and Epidemiology and Prevention. Circulation. 2003;108:2154-2169. doi:10.1161/01.CIR.000009567 6.90936 .80 
4. Hallan SI, Matsushita K, Sang Y, et al.; Chronic Kidney Disease Prognosis Consortium. Age and association of kidney measures with mortality and end-stage renal disease. JAMA. 2012;308:2349-2360. doi:10.1001/jama.2012.16817

5. Collins AJ. Cardiovascular mortality in end-stage renal disease. Am J Med Sci. 2003;325:163-167. doi:10.1097/00000441-200304000-00002

6. Nakamura M, Yamashita T, Yajima J, et al. Impact of reduced renal function on prognosis in Japanese patients with coronary artery disease: a prospective cohort of Shinken Database 2007. Hypertens Res. 2009;32:920-926. doi:10.1038/hr.2009.114

7. Ying-Chun M, Zuo L, Chen J-H, et al; on behalf of the Chinese eGFR Investigation Collaboration. Modified glomerular filtration rate estimating equation for Chinese patients with chronic kidney disease. J Am Soc Nephrol. 17;2006:2937-2944. doi:10.1681/ASN.2006 040368

8. Avci A, Fidan S, Tabakçı MM, et al. Association between the Gensini score and carotid artery stenosis. Korean Circ J. 2016;46:639-645. doi:10.4070/kcj.2016.46.5.639

9. Prichard S. Risk factors for coronary artery disease in patients with renal failure. Am J Med Sci. 2003;325:209-213. doi:10.1097/ 00000441-200304000-00007

10. Sarnak MJ, Levey AS, Schoolwerth AC, et al.; American Heart Association Councils on Kidney in Cardiovascular Disease, High Blood Pressure Research, Clinical Cardiology, and Epidemiology and Prevention. Kidney disease as a risk factor for development of cardiovascular disease: a statement from the American Heart Association Councils on Kidney in Cardiovascular Disease, High Blood Pressure Research, Clinical Cardiology, and Epidemiology and Prevention. Hypertension. 2003;42:1050-1065. doi:10.1161/01. HYP.0000102971.85504.7c

11. McCullough PA, Li S, Jurkovitz CT, et al.; KEEP Investigators. Chronic kidney disease, prevalence of premature cardiovascular disease, and relationship to short-term mortality. Am Heart J. 2008;156:277-283. doi:10.1016/j.ahj.2008.02.024
12. Manjunath G, Tighiouart H, Ibrahim H, et al. Level of kidney function as a risk factor for atherosclerotic cardiovascular outcomes in the community. J Am Coll Cardiol. 2003;41:47-55. doi:10.1016/s07351097(02)02663-3

13. Tonelli M, Jose P, Curhan G, Sacks F, Braunwald E, Pfeffer M; Cholesterol and Recurrent Events (CARE) Trial Investigators. Proteinuria, impaired kidney function, and adverse outcomes in people with coronary disease: analysis of a previously conducted randomised trial. BMJ. 2006;332:1426. doi:10.1136/bmj.38814.566019.2F

14. Liu H, Yu J, Chen F, Li J, Hu D. Inpatients with coronary heart disease have a high prevalence of chronic kidney disease based on estimated glomerular filtration rate (eGFR) in China. Heart Vessels. 2007;22:223-228. doi:10.1007/s00380-006-0964-7

15. Ryoo JH, Kim SG, Suh BS, Kim DI, Park SK. Relationship between chronic kidney disease and risk of coronary heart disease in Korean men. J Korean Med Sci. 2011;26:753-758. doi:10.3346/jkms.201 1.26.6.753

16. Agrawal H, Aggarwal K, Littrell R, et al. Pharmacological and non pharmacological strategies in the management of coronary artery disease and chronic kidney disease. Curr Cardiol Rev. 2015;11 (3):261-269. doi:10.2174/1573403X1103150514155757

17. Manjunath G, Tighiouart H, Coresh J, et al. Level of kidney function as a risk factor for cardiovascular outcomes in the elderly. Kidney Int. 2003;63:1121-1129. doi:10.1046/j.1523-1755.2003.00838.x

18. Mann JF, Gerstein HC, Pogue J, Bosch J, Yusuf S. Renal insufficiency as a predictor of cardiovascular outcomes and the impact of ramipril: the HOPE randomized trial. Ann Intern Med. 2001;134:629-636. doi:10.7326/0003-4819-134-8-200104170-00007
International Journal of General Medicine

\section{Publish your work in this journal}

The International Journal of General Medicine is an international, peer-reviewed open-access journal that focuses on general and internal medicine, pathogenesis, epidemiology, diagnosis, monitoring and treatment protocols. The journal is characterized by the rapid reporting of reviews, original research and clinical studies

\section{Dovepress}

across all disease areas. The manuscript management system is completely online and includes a very quick and fair peer-review system, which is all easy to use. Visit http://www.dovepress.com/ testimonials.php to read real quotes from published authors. 